\title{
INDICAÇÕES DO USO DE MEMBRANA DE OXIGENAÇÃO EXTRACORPÓREA EM PACIENTES COM COVID-19
}

\section{ARTIGO DE REVISÃO}

GUERRA, Marcela Scardua Zanotti ${ }^{1}$, REIS, Alexandre Ramos Furtado², DANTAS, Ellen Karoliny de Oliveira ${ }^{3}$, SANTOS, Helen Amanda Pinto dos ${ }^{4}$, SANTOS, Hugo Leonardo Ribeiro Canuto5, DUARTE, Karoline Simões Baldotto ${ }^{6}$, SANGUINO, Larissa de Oliveira7 ${ }^{7}$ CHASTINET, Maria Beatriz Aguiar ${ }^{8}$, ARAUJO, Matheus Maia de $^{9}$, OLIVEIRA, Paula Fernanda Patriolina Teixeira de ${ }^{10}$, SOUZA, Victoria Tamay de $^{11}$, OLIVEIRA, Igor de Sousa ${ }^{12}$, RIBEIRO, Yana Sarah Fernandes Souza ${ }^{13}$, VASCONCELOS, Gilberto Loiola de ${ }^{14}$

GUERRA, Marcela Scardua Zanotti. Et al. Indicações do uso de membrana de oxigenação extracorpórea em pacientes com COVID-19. Revista Científica Multidisciplinar Núcleo do Conhecimento. Ano 06, Ed. 07, Vol. 12, pp. 05-21. Julho de 2021. ISSN: 2448-0959, Link de acesso: https://www.nucleodoconhecimento.com.br/saude/indicacoes-do-uso, 10.32749/nucleodoconhecimento.com.br/saude/indicacoes-do-uso

\footnotetext{
${ }^{1}$ Discente do curso de Medicina do Centro Universitário do Espírito Santo.

${ }^{2}$ Discente do curso de Medicina do Centro Universitário UNIFACID.

${ }^{3}$ Discente do curso de Medicina do Centro Universitário do Espírito Santo.

${ }^{4}$ Discente do curso de Saúde Coletiva da Universidade Federal do Oeste do Pará - UFOPA.

${ }^{5}$ Discente do curso de Medicina do Instituto Master de Ensino Presidente Antônio Carlos - IMEPAC.

${ }^{6}$ Discente do curso de Medicina do Centro Universitário do Espírito Santo.

${ }^{7}$ Discente do curso de Medicina da Universidade Nilton Lins.

${ }^{8}$ Discente do curso de Medicina do Centro Universitário INTA - UNINTA.

${ }^{9}$ Discente do curso de Medicina do Centro Universitário INTA - UNINTA.

${ }^{10}$ Discente do curso de Medicina da Universidade Federal do Ceará - Campus Sobral.

${ }^{11}$ Discente do curso de Medicina da Escola Superior de Ciências da Saúde.

12 Discente do curso de Medicina da Universidade Federal de Campina Grande, Centro de Formação dos Professores, Cajazeiras - Paraíba.

${ }^{13}$ Discente do curso de Medicina do Centro Universitário INTA - UNINTA.

${ }^{14}$ Orientador. Médico residente em Clínica Médica, formado pela Universidade Federal do Ceará.
}

RC: 92267

Disponível em: https://www.nucleodoconhecimento.com.br/saude/indicacoes-do-uso 


\section{RESUMO}

Objetivo: Esclarecer as principais indicações de uso terapêutico do ECMO (Oxigenação por Membrana Extracorpórea) em pacientes portadores do vírus SarsCoV-2. Métodos: Esta pesquisa configura revisão bibliográfica do tipo integrativa baseada na análise de 11 artigos disponíveis no Portal da Biblioteca Virtual em Saúde (BVS) e em seus sites aliados, mediante aplicação de fórmula de busca pautada no vocabulário disponível no DeCS (Descritores em Ciências da Saúde). Resultados: Dentre os 11 estudos selecionados, 36,36\% ( $n=4)$ evidenciaram que a principal indicação para o uso de oxigenação por membrana extracorpórea (ECMO) em pacientes com COVID-19 é a presença de hipoxemia refratária aos suportes respiratórios padronizados, tais como ventilação mecânica protetora pulmonar, recrutamento pulmonar, posicionamento prono, bloqueio neuromuscular e sedação. No que se refere ao uso da ECMO em pacientes portadores de Sars-CoV-2, 18,18\% $(n=2)$ dos artigos abordaram o uso da técnica citada em pacientes pediátricos e atestaram, em relação às indicações, que a grande maioria das diretrizes hospitalares utiliza as mesmas indicações para ECMO em pacientes com COVID-19 e para outras doenças virais, quais sejam: hipoxemia refratária e agravamento da hipercapnia, SDRA (Síndrome do Desconforto Respiratório Agudo) ou necessidade contínua de drogas vasoativas secundárias ao agravamento do quadro viral. Conclusão: Estudos em ambiente de terapia intensiva demonstraram taxas de mortalidade discretamente menores em pacientes com SDRA por infecção pelo COVID-19 que fizeram uso de ECMO, em comparação à terapia respiratória convencional. A indicação de ECMO em pacientes pediátricos com COVID- 19 segue a mesma recomendação dos casos de outros quadros virais e seu uso pode variar entre 14-21 dias, apesar de controvérsias.

Palavras-chave: Infecções por coronavírus, Oxigenação por Membrana Extracorpórea, ECMO.

RC: 92267

Disponível em: https://www.nucleodoconhecimento.com.br/saude/indicacoes-do-uso 


\section{INTRODUÇÃO}

A oxigenação por membrana extracorpórea (ECMO) é uma medida terapêutica que possibilita substituição temporária de funções cardiopulmonares a fim de manter perfusão tecidual e oxigenação sanguínea em pacientes com declínio funcional desses sistemas refratários ao manejo clínico (XIAOCHUN et al., 2020) (CHAVES et al., 2019).

Composto essencialmente por uma bomba de propulsão, membrana oxigenadora, cânulas de drenagem e retorno sanguíneo e um sistema de controle térmico, o dispositivo de ECMO pode ser configurado em duas modalidades: ECMO venovenosa (ECMO-VV) e ECMO venoarterial (ECMO-VA). A ECMO-VV é a modalidade de escolha em pacientes com quadro de insuficiência respiratória hipoxêmica ou insuficiência respiratória hipercápnica com função cardíaca preservada ou pouco reduzida. Já a ECMO-VA, é a opção de escolha em pacientes que apresentam falência cardíaca associada ou não a declínio funcional pulmonar (CHAVES et al., 2019).

A emergência da doença COVID-19, causada pelo coronavírus da Síndrome Respiratória Aguda Grave 2 (SARS-CoV-2), pontua a ECMO como uma alternativa terapêutica para pacientes com insuficiência respiratória grave. De acordo com a Organização Mundial de Saúde (2021), em seu Guia de Manejo Clínico à COVID-19 considera-se uso do dispositivo em pacientes graves que apresentem quadro de hipoxemia refratária à ventilação protetiva e estejam internados em unidade de saúde com especialistas em ECMO. Nesse sentido, o guia refere provável redução da mortalidade em pacientes com Síndrome Respiratória Aguda Grave (SRAG) submetidos à ECMO, baseado em uma análise bayesiana de dados de um ensaio clínico randomizado que avaliou a oxigenação extracorpórea precoce comparada à ventilação convencional em pacientes com SRAG. Porém, é evidenciado que estudos futuros precisam ser realizados e no presente as indicações de ECMO em pacientes com SRAG seguem individualizadas.

RC: 92267

Disponível em: https://www.nucleodoconhecimento.com.br/saude/indicacoes-do-uso 
Nesse contexto, a ECMO atua como alternativa de alívio a lesões pulmonares causadas por ventilação mecânica prolongada, assim como promove otimização da oxigenação em pacientes com pneumopatias graves não responsivas à ventilação mecânica exclusiva (MARHONG, et al., 2015). Frente à pandemia da COVID-19, a ECMO-VV é aplicada na maioria dos casos, porém alguns pacientes beneficiam-se do uso de ECMO-VA, principalmente devido a distúrbios hemodinâmicos secundários à infecção pelo vírus ou lesões cardíacas primárias como arritmias ou miocardites (WIEDEMANN et al., 2020).

Ao propor o uso de ECMO, deve-se individualizar os riscos e benefícios que tal intervenção traz ao paciente. Entre as principais complicações da ECMO estão: distúrbios de coagulação induzidos pelo dispositivo, desregulação de trocas gasosas e infecções no circuito. (XIAOCHUN; et al., 2020) Por esse motivo, a Organização de Suporte de Vida Extracorpórea (ELSO), determinou algumas contraindicações ao uso de ECMO, entre elas estão: pacientes com doença terminal, danos graves ao sistema nervoso central, comorbidades significativas e ventilação mecânica por um período superior a 7 dias. Logo, nessas situações os riscos superariam os benefícios do uso do dispositivo (SHEKAR et al., 2020).

Desse modo, percebe-se que o momento do suporte de ECMO e a seleção do paciente são cruciais para o resultado, uma vez que está relacionado diretamente com as condições homeostáticas e prognósticas do paciente (W.D. et al., 2020). Desse modo, emerge a questão: "Quais seriam as indicações mais significativas do uso do ECMO em COVID-19 no manejo de pacientes críticos?".

Com isso, é sabido que a gravidade da COVID-19 está ocasionando diversas mortes no mundo todo, com isso a oxigenação por membrana extracorpórea (ECMO) representa uma opção terapêutica valiosa em pacientes com insuficiência cardíaca ou pulmonar grave. Embora apenas uma proporção restrita de pacientes com COVID-19 evoluiu com insuficiência respiratória ou cardíaca refratária ao tratamento preconizado, é de extrema importância definir claramente os critérios para o uso de ECMO nesta população de pacientes em constante crescimento.

RC: 92267

Disponível em: https://www.nucleodoconhecimento.com.br/saude/indicacoes-do-uso 
Dado que o uso de terapia por membrana extracorpórea (ECMO) vêm evidenciando benefícios, tal comportamento pode favorecer uma maior chance de recuperação de pacientes com insuficiência respiratória e circulatória grave, e até minimizar o avanço de intercorrências paralelas a esse desfecho.

Por fim, evidencia-se que o objetivo principal deste estudo justamente é esclarecer os principais pontos de indicações que estão relacionados à conduta terapêutica do uso do ECMO diante da gravidade dos pacientes diagnosticados com COVID-19.

\section{METODOLOGIA}

O presente artigo é um estudo pautado em uma revisão bibliográfica do tipo integrativa, com abordagem quanti-qualitativa, de natureza aplicada que busca mostrar as indicações do uso da membrana extracorpórea na COVID-19 e, desse modo, colaborar com a ampliação do conhecimento sobre sua aplicabilidade. Ademais, ressalta-se o objetivo descritivo e procedimento bibliográfico.

A base para a pesquisa formulou-se no Portal da Biblioteca Virtual em Saúde (BVS) e em seus sites aliados. Além disso, salienta-se que o levantamento e seleção dos artigos foram feitos no mês de abril de 2021. Diante disso, foi realizada a escolha de descritores pertinentes à temática presente para esse estudo, por meio do vocabulário disponível no DeCS (Descritores em Ciências da Saúde). Tais descritores são: "oxigenação por membrana extracorpórea" e "infecção por coronavírus", sendo utilizados filtros em português, inglês e espanhol, e também seus sinônimos pertinentes, que associados possibilitaram uma melhor e mais ampla busca e seleção dos artigos. Além disso, foram usados os seguintes operadores booleanos: parênteses, AND e OR.

Dessa forma, foram encontrados um total de 28 artigos na base de dados citada acima, sendo aplicados alguns critérios de inclusão e exclusão. Salienta-se que foram elegíveis os artigos que elucidavam pacientes, sendo crianças ou adultos, que apresentou uso da oxigenação por membrana extracorpórea e diagnóstico

RC: 92267

Disponível em: https://www.nucleodoconhecimento.com.br/saude/indicacoes-do-uso 
confirmado de COVID-19, independente de etnia ou sexo, artigos disponíveis na integra, artigos disponíveis em espanhol, inglês e português e artigos a partir de 2019. Por outro lado, os critérios de exclusão usados foram: artigos que abordavam a oxigenação extracorpórea, mas não havia diagnóstico confirmado de COVID-19, artigos de opinião, metanálises, revisões sistemáticas, estudos duplicados e artigos que não estavam disponíveis na integra.

Com isso, foi feita uma análise de busca por dois pesquisadores, ISO e EKOD, que avaliaram todos os títulos e resumos dos artigos e selecionaram os estudos baseados nos critérios de elegibilidade previamente citados. Nesse momento, dos 28 artigos previamente encontrados, restaram-se selecionados 11 artigos na Biblioteca Virtual em Saúde (BVS).

Desse modo, é importante salientar que não ocorreu submissão ao Comitê de Ética em Pesquisa (CEP), visto não ser necessário, uma vez que este não tem aplicação prática, sendo então dispensada a submissão.

Por fim, a análise de dados irá ocorrer por meio da apresentação de um quadro com aspectos técnicos dos artigos, contendo as principais informações, sendo essas: título, ano e local de publicação, autoria e principais resultados.

\section{RESULTADOS}

\begin{tabular}{|c|c|c|c|}
\hline & Título & $\begin{array}{l}\text { Autores, ano } \\
\text { de publicação } \\
\text { e país }\end{array}$ & Principais Resultados \\
\hline ARTIGO I & $\begin{array}{l}\text { Extracorporeal } \\
\text { Membrane } \\
\text { Oxygenation } \\
\text { (ECMO) in } \\
\text { Critically }\end{array}$ & $\begin{array}{l}\text { MA et al., 2020, } \\
\text { China. }\end{array}$ & $\begin{array}{l}\text { A ECMO constitui modalidade } \\
\text { terapêutica de extrema } \\
\text { relevância para pacientes } \\
\text { portadores de Sars-CoV-2, uma } \\
\text { vez que melhora o prognóstico }\end{array}$ \\
\hline
\end{tabular}

RC: 92267

Disponível em: https://www.nucleodoconhecimento.com.br/saude/indicacoes-do-uso 


\begin{tabular}{|c|c|c|c|}
\hline & $\begin{array}{l}\text { Patients with } \\
\text { Coronavirus } \\
\text { Disease } 2019 \\
\text { (COVID-19) } \\
\text { Pneumonia and } \\
\text { Acute Respiratory } \\
\text { Distress } \\
\text { Syndrome } \\
\text { (ARDS). }\end{array}$ & & $\begin{array}{l}\text { de indivíduos com hipoxemia } \\
\text { refratária. }\end{array}$ \\
\hline $\begin{array}{l}\text { ARTIGO } \\
\text { II }\end{array}$ & $\begin{array}{l}\text { Extracorporeal } \\
\text { Life Support } \\
\text { Organization } \\
\text { Coronavirus } \\
\text { Disease } 2019 \\
\text { Interim Guidelines: } \\
\text { A Consensus } \\
\text { Document from an } \\
\text { International } \\
\text { Group } \\
\text { Interdisciplinary } \\
\text { Extracorporeal } \\
\text { Membrane } \\
\text { Oxygenation } \\
\text { Providers. }\end{array}$ & $\begin{array}{l}\text { RAMAN et al., } \\
2020 \text {, Estados } \\
\text { Unidos. }\end{array}$ & $\begin{array}{l}\text { Para um bom fornecimento da } \\
\text { ECMO faz-se necessário um } \\
\text { planejamento de suporte às } \\
\text { instituições nas quais a } \\
\text { tecnologia está disponível. }\end{array}$ \\
\hline $\begin{array}{l}\text { ARTIGO } \\
\text { III }\end{array}$ & $\begin{array}{l}\text { Recommendations } \\
\text { for extracorporeal } \\
\text { membrane } \\
\text { oxygenation } \\
\text { (ECMO) in } \\
\text { COVID-19 }\end{array}$ & $\begin{array}{l}\text { WIEDEMANN } \\
\text { et al., 2020, } \\
\text { Áustria. }\end{array}$ & $\begin{array}{l}\text { Observou-se que a ECMO é } \\
\text { uma opção para pacientes com } \\
\text { insuficiência cardíaca ou } \\
\text { pulmonar grave. Neste cenário, } \\
\text { o estudo discute os critérios } \\
\text { clínicos para o uso adequado }\end{array}$ \\
\hline
\end{tabular}

RC: 92267

Disponível em: https://www.nucleodoconhecimento.com.br/saude/indicacoes-do-uso 
patients.

da ECMO em pacientes com COVID-19.

\begin{tabular}{|c|c|c|c|}
\hline ARTIGO & $\begin{array}{l}\text { Extracorporeal } \\
\text { Membrane } \\
\text { Oxygenation for } \\
\text { Coronavirus } \\
\text { Disease 2019- } \\
\text { Induced Acute } \\
\text { Respiratory } \\
\text { Distress } \\
\text { Syndrome: } \\
\text { Multicenter } \\
\text { Descriptive Study. }\end{array}$ & $\begin{array}{l}\text { YANG et al., } \\
2020 \text {, Estados } \\
\text { Unidos. }\end{array}$ & $\begin{array}{l}\text { A ECMO é eficaz em pacientes } \\
\text { portadores de COVID-19, } \\
\text { graves, e que apresentam } \\
\text { pneumonia e síndrome do } \\
\text { desconforto respiratório agudo } \\
\text { grave. }\end{array}$ \\
\hline ARTIGO & $\begin{array}{l}\text { Extracorporeal } \\
\text { Membrane } \\
\text { Oxygenation for } \\
\text { Pediatric Patients } \\
\text { With Coronavirus } \\
\text { Disease 2019- } \\
\text { Related Illness. }\end{array}$ & $\begin{array}{l}\text { MACGREGOR } \\
\text { et al., 2020, } \\
\text { Estados } \\
\text { Unidos. }\end{array}$ & $\begin{array}{l}\text { A maior parte dos centros } \\
\text { pediátricos aceitou o uso da } \\
\text { ECMO em pacientes } \\
\text { pediátricos com complicações } \\
\text { referentes à COVID-19. }\end{array}$ \\
\hline $\begin{array}{l}\text { ARTIGO } \\
\text { VI }\end{array}$ & $\begin{array}{l}\text { Guidance for the } \\
\text { management of } \\
\text { adult patients with } \\
\text { coronavirus } \\
\text { disease } 2019 \text {. }\end{array}$ & $\begin{array}{l}\text { QU et al., 2020, } \\
\text { China. }\end{array}$ & $\begin{array}{l}\text { O estudo fornece diretrizes de } \\
\text { manejo de indivíduos com } \\
\text { COVID-19 e atesta que a } \\
\text { ECMO é benéfica no } \\
\text { tratamento de pacientes com } \\
\text { SDRA. }\end{array}$ \\
\hline $\begin{array}{l}\text { ARTIGO } \\
\text { VII }\end{array}$ & $\begin{array}{l}\text { The role of } \\
\text { extracorporeal life }\end{array}$ & $\begin{array}{l}\text { SULTAN et al., } \\
\text { 2020, Estados }\end{array}$ & $\begin{array}{l}\text { A utilização de ECMO exige } \\
\text { habilidades }\end{array}$ \\
\hline
\end{tabular}

RC: 92267

Disponível em: https://www.nucleodoconhecimento.com.br/saude/indicacoes-do-uso 


\begin{tabular}{|c|c|c|c|}
\hline & $\begin{array}{l}\text { support for } \\
\text { patients with } \\
\text { COVID-19: } \\
\text { Preliminary results } \\
\text { from a statewide } \\
\text { experience. }\end{array}$ & Unidos. & $\begin{array}{l}\text { treinamento especializado e } \\
\text { recursos tecnológicos. Tal } \\
\text { abordagem terapêutica não é } \\
\text { isenta de complicações e, } \\
\text { dentre as quais, pode-se citar } \\
\text { trombose, hemorragia e } \\
\text { disseminação infecciosa. }\end{array}$ \\
\hline $\begin{array}{l}\text { ARTIGO } \\
\text { VIII }\end{array}$ & $\begin{array}{l}\text { Planning and } \\
\text { provision of } \\
\text { ECMO services } \\
\text { for severe ARDS } \\
\text { during the COVID- } \\
19 \text { pandemic and } \\
\text { other outbreaks of } \\
\text { emerging } \\
\text { infectious } \\
\text { diseases. }\end{array}$ & $\begin{array}{l}\text { RAMANATHAN } \\
\text { et al., 2020, } \\
\text { Estados } \\
\text { Unidos. }\end{array}$ & $\begin{array}{l}\text { O estudo expõe a importância } \\
\text { de compreender melhor a } \\
\text { fisiopatologia associada ao } \\
\text { desenvolvimento de síndrome } \\
\text { do desconforto respiratório } \\
\text { agudo (SDRA) porque isto } \\
\text { facilitaria o uso correto da } \\
\text { ECMO em períodos de surtos. }\end{array}$ \\
\hline $\begin{array}{l}\text { ARTIGO } \\
\text { IX }\end{array}$ & $\begin{array}{l}\text { Extracorporeal } \\
\text { membrane } \\
\text { oxygenation } \\
\text { support in } 2019 \\
\text { novel coronavirus } \\
\text { disease: } \\
\text { indications, timing, } \\
\text { and } \\
\text { implementation. }\end{array}$ & $\begin{array}{l}\text { LI et al., 2020, } \\
\text { China }\end{array}$ & $\begin{array}{l}\text { A letalidade dos pacientes que } \\
\text { receberam a ECMO foi menor } \\
\text { do que os indivíduos que } \\
\text { receberam apenas cuidados } \\
\text { respiratórios convencionais. }\end{array}$ \\
\hline $\begin{array}{l}\text { ARTIGO } \\
X\end{array}$ & $\begin{array}{l}\text { Optimizing } \\
\text { provision of } \\
\text { extracorporeal life } \\
\text { support during the }\end{array}$ & $\begin{array}{l}\text { PARHAR et al., } \\
\text { 2020, Canadá. }\end{array}$ & $\begin{array}{l}\text { Indica-se o suporte de vida } \\
\text { extracorpórea (ECLS) para } \\
\text { pacientes com hipoxemia }\end{array}$ \\
\hline
\end{tabular}

RC: 92267

Disponível em: https://www.nucleodoconhecimento.com.br/saude/indicacoes-do-uso 


\begin{tabular}{|c|c|c|c|}
\hline & $\begin{array}{l}\text { COVID-19 } \\
\text { pandemic: } \\
\text { practical } \\
\text { considerations for } \\
\text { Canadian } \\
\text { jurisdictions }\end{array}$ & & refratária. \\
\hline $\begin{array}{l}\text { ARTIGO } \\
\text { XI }\end{array}$ & $\begin{array}{ll}\text { Membrana } & \text { de } \\
\text { oxigenación } & \\
\text { extracorpórea } & \text { en } \\
\text { infección } & \text { por } \\
\text { COVID-19. } & \end{array}$ & $\begin{array}{l}\text { IECS - Instituto } \\
\text { de Efectividad } \\
\text { Clinica y } \\
\text { Sanitária, } \\
2020, \\
\text { Argentina. }\end{array}$ & $\begin{array}{l}\text { O uso da membrana de } \\
\text { oxigenação extracorpórea } \\
\text { (ECMO) é indicado em } \\
\text { pacientes com hipoxemia } \\
\text { refratária e, nestes indivíduos, } \\
\text { observa-se uma redução na } \\
\text { mortalidade após o uso da } \\
\text { abordagem terapêutica de } \\
\text { suporte circulatório. }\end{array}$ \\
\hline
\end{tabular}

Fonte: Autoria Própria, 2021.

Dentre os 11 estudos selecionados, $36,36 \%(n=4)$ evidenciaram que a principal indicação para o uso ECMO em pacientes com COVID-19 é a presença de hipoxemia refratária ao suporte respiratório padrão, tal como ventilação mecânica protetora pulmonar, recrutamento pulmonar, posicionamento prono, bloqueio neuromuscular e sedação (MA et al., 2020). Considerando-se que a ECMO é uma tecnologia cara e escassa, principalmente no atual cenário de pandemia, torna-se essencial à delimitação deste recurso a pacientes com hipoxemia refratária, para que o mesmo esteja disponível quando necessário (PARHAR et al., 2020). Tal medida terapêutica auxiliaria a respiração e a circulação desses pacientes, contribuindo para uma significativa redução na mortalidade (IECS - INSTITUTO DE EFECTIVIDAD CLINICA Y SANITÁRIA, 2020).

RC: 92267

Disponível em: https://www.nucleodoconhecimento.com.br/saude/indicacoes-do-uso 
Em contrapartida, 36,36\% dos estudos $(n=4)$ apontaram a presença da Síndrome do Desconforto Respiratório Agudo (SDRA) grave como condição na qual a utilização de ECMO seria eficaz no tratamento de pacientes infectados pelo Sars-CoV-2. O estudo de Ramanathan et al. (2020) sugere que pacientes com COVID-19 que desenvolvem SDRA grave e não se beneficiam do tratamento convencional podem obter sucesso com a ECMO veno-venosa.

Dentre os ensaios que abordam a SDRA grave como indicação de ECMO, alguns mencionam valores de corte em determinados parâmetros (após tratamento com suporte respiratório padrão) para determinar a aplicação dessa opção terapêutica, tais como relação entre $\mathrm{PaO} 2$ (Pressão Arterial de Oxigênio) e FiO2 (Fração Inspirada de Oxigênio) menor que $50 \mathrm{mmHg}$ por mais de 3 horas, menor que 80 $\mathrm{mmHg}$ por mais de 6 horas, ou a presença de um pH menor que 7,25 com PaCO2 (Pressão Arterial de Dióxido de Carbono) maior ou igual a $60 \mathrm{mmHg}$ por mais de seis horas (WIEDEMANN et al., 2020; QU et al., 2020). Ademais, o estudo de Wiedemann et al. (2020) destaca algumas situações que representam contraindicações absolutas ao emprego de ECMO em pacientes com COVID-19: é o caso daqueles com mais de 75 anos de idade, com doenças em estágio terminal (em que a expectativa de vida é inferior a 1 ano), com déficit neurológico grave préexistente ou câncer disseminado.

Em relação à efetividade do uso de ECMO no tratamento de pacientes com COVID19 e SDRA grave, é válido destacar o estudo descritivo e multicêntrico de Yang et al. (2020). Na pesquisa, foram analisados 129 pacientes com SDRA grave decorrente da infecção pelo Sars-CoV-2, internados na unidade de terapia intensiva (UTI) de dois hospitais chineses de referência. Cinquenta e nove pacientes receberam ventilação mecânica e vinte e um deles receberam suporte de oxigenação por membrana extracorpórea. Houve uma pequena diferença na mortalidade observada entre os dois grupos: $63,2 \%$ dos pacientes submetidos à ventilação mecânica sofreram óbito, ao passo que esta porcentagem foi de $57,1 \%$ para os pacientes em uso de ECMO. O estudo demonstrou, ainda, que a presença de retenção grave de 
CO2 e acidose antes da aplicação do ECMO foi indicativa de pior prognóstico, estando estas condições relacionadas à maior chance de morte (YANG et al., 2020).

Também em relação ao suporte de oxigenação por membrana extracorpórea na nova doença coronavírus em um estudo feito no Hospital Wuhan Jinyintan, observou-se que a SDRA foi relatada em 12 (29\%) entre 41 pacientes confirmados. Dentre os 41 pacientes, 13 pacientes receberam atendimento médico na unidade de terapia intensiva (UTI), 4 pacientes receberam ventilação mecânica invasiva, enquanto em dois pacientes foi aplicado tratamento de oxigenação por membrana extracorpórea (ECMO). Finalmente, seis dos 41 pacientes morreram. Os dados clínicos de 99 pacientes confirmados do mesmo hospital demonstraram que 17 em 99 pacientes desenvolveram SDRA; entre eles, três receberam tratamento com ECMO e 11 morreram (LI et al., 2020).

No que se refere ao uso da ECMO em pacientes portadores de Sars-CoV-2, 18,18\% $(n=2)$ dos artigos abordaram o uso da técnica citada em pacientes pediátricos e atestaram, em relação às indicações, que a grande maioria das diretrizes hospitalares utiliza as mesmas indicações para ECMO em pacientes com COVID-19 e para outras doenças virais, quais sejam: hipoxemia refratária e agravamento da hipercapnia, SDRA ou necessidade contínua de drogas vasoativas secundárias ao agravamento do quadro viral (SHEKAR et al., 2020). Além disso, todos os programas oferecem ECMO veno-venosa e a maioria oferece ECMO venoarterial (MACGREGOR et al., 2020).

No que tange ao tempo, estudo realizado em diversos centros hospitalares neonatais e pediátricos dos EUA evidenciou que entre as instituições com diretrizes estabelecidas de indicações de ECMO para doenças relacionadas à COVID-19, apenas três (6\%) limitam o número máximo de dias de uso do suporte circulatório (variação de 14 a 21 dias). No entanto, ainda se trata de decisão controversa, visto que em outras situações clínicas, como em execuções estendidas de ECMO venovenosa para insuficiência respiratória pediátrica, o tempo necessário de aplicação é maior (MACGREGOR et al., 2020).

RC: 92267

Disponível em: https://www.nucleodoconhecimento.com.br/saude/indicacoes-do-uso 
No que se refere à prioridade na alocação de recursos, estudo de MacGregor et al. (2020) indica que, embora especialistas concordem que a preferência da utilização de modalidades terapêuticas escassas deve ser direcionada aos pacientes que receberão o maior benefício, permanece uma discordância significativa entre o alcance dos objetivos: "salvar o máximo de vidas individuais" ou "salvar o máximo de anos de vida". Neste sentido, a ênfase no maior número de vidas individuais salvas priorizaria aqueles com maior probabilidade de sobreviver ao tratamento, independentemente da idade. Pesquisas anteriores de envolvimento da comunidade demonstram uma ampla gama de aceitabilidade do uso de vários princípios orientadores, como "priorizar aqueles que viveram menos estágios da vida" ao determinar a alocação de recursos escassos durante um desastre (MACGREGOR et al., 2020).

\section{DISCUSSÃO}

O surgimento da COVID-19, e, consequentemente, o aumento de internações hospitalares, acionou um alerta mundial na busca por possíveis tratamentos. Por se tratar de uma doença sistêmica que está afetando de forma grave a população fazse necessário medidas de suporte frente a diminuição da capacidade respiratória, níveis de oxigenação e demais complicações dos pacientes acometidos. Nesse sentido, se tem utilizado, como alternativa, para a melhora desses quadros críticos o uso da oxigenação por membrana extracorpórea (ECMO).

A ECMO é uma tecnologia que emprega dispositivos artificiais para imitar a função cardiopulmonar mantendo o suprimento de sangue e trocas gasosas por todo o corpo. Alguns protocolos indicam o uso de ECMO como terapia de resgate após a falha do tratamento de suporte, outros autores sugerem o início precoce de ECMO na SDRA, ou seja, antes do MODS ou lesão pulmonar grave, apresentando resultados benéficos, principalmente em pacientes jovens. Porém, devido à falta de equipamentos, especialista e do alto custo do procedimento, a ECMO precoce se torna algo impraticável (MA et al., 2020).

RC: 92267

Disponível em: https://www.nucleodoconhecimento.com.br/saude/indicacoes-do-uso 
A ECMO foi considerada uma opção eficaz durante o tratamento dos surtos de influenza H1N1 em 2009. Em relação ao COVID-19, ainda não está claro se trará resultados satisfatórios, porém já se sabe que a ECMO pode auxiliar as trocas gasosas em pacientes com síndrome do desconforto respiratório agudo (SDRA) e estar associado a uma mortalidade mais baixa, tornando-se um tratamento de resgate eficaz (YANG et al., 2020).

A maioria dos pacientes contaminados com COVID-19, mesmo desenvolvendo pneumonia, se recuperaram e tiveram sintomas brandos. Porém, alguns pacientes progrediram rapidamente para síndrome da angústia respiratória aguda (SDRA) e choque séptico, lesão miocárdica, lesão renal aguda (LRA) e outras disfunções orgânicas podem ocorrer em pacientes com doença grave. Nesses casos mais graves, a ECMO é frequentemente indicada no resgate de pacientes com COVID19. As informações sobre ECMO no COVID-19 devem ser compartilhadas para que se possa sanar as lacunas desse procedimento (LI et al., 2020) (QU et al., 2020).

Pessoas de todas as idades são suscetíveis à infecção pelo COVI-19, porém adultos idosos e pessoas com comorbidades, como doença pulmonar obstrutiva crônica, diabetes, hipertensão e doenças cardíacas, apresentam risco aumentado. Além disso, contatos próximos com pacientes infectados e a equipe médica também possuem maior risco de infecção. O período de incubação é de 1 a 14 dias e as manifestações clínicas iniciais são: início agudo de febre e fadiga e tosse seca. Uma minoria de pacientes apresenta: congestão nasal, coriza, dor de garganta, dores musculares e diarreia. Em casos mais graves ocorre rigidez do tórax e dispneia. Em pacientes críticos pode ocorrer SDRA, choque séptico e acidose metabólica. Nos estágios iniciais, a imagem do tórax apresenta pequenas sombras irregulares e alterações intersticiais na zona periférica dos pulmões que se desenvolvem em vidro fosco. Em casos graves pode ocorrer consolidação pulmonar (QU et al., 2020).

Em relação aos pacientes pediátricos, $58 \%$ dos centros de ECMO aprovaram diretrizes para o uso de suporte de ECMO nesses pacientes com doenças relacionadas ao COVID-19, sendo afirmado em $94 \%$ dos centros que as indicações

RC: 92267

Disponível em: https://www.nucleodoconhecimento.com.br/saude/indicacoes-do-uso 
de ECMO para COVID-19 eram semelhantes às usadas em outras doenças virais. Além disso, as diretrizes restringem o uso de ECMO em pacientes pediátricos com as seguintes patologias: doença terminal, danos graves do SNC, status de DNR, falência de órgãos multissistêmicos, ventilação mecânica prolongada por mais de 7 dias e insuficiência renal (MACGREGOR et al., 2020).

A grande demanda das terapias intensivas relacionado ao COVID-19 ocorre devido a insuficiência respiratória hipoxêmica, necessitando de ventilação mecânica. Porém, uma pequena porção desses pacientes apresentou falha nas terapias convencionais e podem precisar de ECMO, e, nesses casos, deve-se considerar a capacidade limitada de oferecer esse suporte. Nesse sentido, recomendam-se critérios de seleção de candidatos mais adequados, como por exemplo, jovens, pacientes com falência de um único órgão, previamente saudáveis, ou seja, pacientes que têm a maior chance de recuperação com esse procedimento.

Deve-se ressaltar que existem contraindicações absolutas, como idade avançada, demência, doença pulmonar avançada, malignidade disseminada, doença vascular periférica grave, desnutrição proteico-energética, diabetes não controlado com disfunção crônica de órgão alvo, lesão neurológica aguda, contraindicação para anticoagulação, incapacidade de aceitar hemoderivados e RCP em curso (RAMAN et al., 2020).

Segundo Qu et al. (2020), a ECMO deve ser aplicado de acordo com as seguintes diretrizes: (i) $\mathrm{PaO} 2$ / FiO2 600 mmHg; (ii) frequência de ventilação> 35 vezes/min enquanto $\mathrm{pH}<7,2$, e pressão de platô $30 \mathrm{cmH} 2 \mathrm{O}$; (iii) idade do paciente $<70$ anos; (iv) duração de ventilação mecânica $<7$ dias. Também pode ser aplicado nas seguintes condições: (i) $\mathrm{PaO} 2 / \mathrm{FiO} 23$ h; (ii) $\mathrm{PaO} 2 / \mathrm{FiO} 26$ h; ou (iii) $\mathrm{pH}$ do sangue arterial $<7,25$ acompanhada por pressão parcial de dióxido de carbono $>60 \mathrm{mmHg}$ por $>6$ h. Além disso, a aplicação antecipada (PaO2 /FiO2 100-150 mmHg) de ECMO no tratamento de SDRA diminui o impulso respiratório, inflamação dos pulmões e de todo o corpo, impedindo o comprometimento do pulmão e de outras funções orgânicas.

RC: 92267

Disponível em: https://www.nucleodoconhecimento.com.br/saude/indicacoes-do-uso 
A ECMO já demonstrou benefícios de sobrevida, sendo utilizado nos tratamentos de infecções graves e influenza H1N1 no ano de 2009. Em relação ao COVID-19 não foi demonstrado respostas devido a falta de evidências clínicas, sendo necessário mais dados para estimar o real benefício da tecnologia. Além disso, considerando a complexidade da ECMO, é importante que as equipes de UTI sejam bem treinadas e aptas a realizar esse procedimento (MA et al., 2020).

Vale ressaltar que a OMS, em seu guia de tratamento clínico da infecção respiratória aguda grave, indicou o uso de ECMO em pacientes com suspeita de COVID-19 e com hipoxemia refratária, desde que o método seja realizado por especialistas em ECMO. Além disso, evidências sugerem que pacientes devidamente selecionados com SRAG e que não tiveram bons resultados com terapias convencionais, podem obter bons resultados com a ECMO veno-venosa (MINISTÉRIO DA SAÚDE, 2020).

Como evidenciado nos resultados, houve uma pequena diferença na mortalidade observada entre os dois grupos: pacientes submetidos à ventilação mecânica e paciente submetidos ECMO. Em comparação com o contexto de SDRA grave fora do contexto do coronavírus, o estudo (EOLIA, 2018) mostrou que a ECMO resultou em melhor oxigenação, com mais dias sem evolução para insuficiência renal aguda, além de 11 por cento de diferença na mortalidade real em 60 dias em comparação a pacientes submetidos a suporte ventilatório tradicional, sendo que a sobrevida foi maior naqueles submetidos à ECMO de forma precoce (até 02 dias do diagnóstico de SDRA grave).

Entretanto, mostrou também maior taxa de sangramentos com necessidade de transfusão e trombocitopenia grave em comparação com a terapia tradicional. Também no (ELSO, 2019), que incluiu 1035 casos no contexto de COVID-19 em mais de 200 hospitais internacionais, o artigo relata que se demonstrou uma mortalidade estimada de 40 por cento após início da ECMO em pacientes com quadro de SDRA grave refratária a medidas conservadoras, resultados que se mostraram consistentes com dados apresentados em nosso artigo até o momento.

RC: 92267

Disponível em: https://www.nucleodoconhecimento.com.br/saude/indicacoes-do-uso 
Levando em consideração o quadro autolimitado e potencialmente reversível do COVID-19, podemos concluir que é essencial integrar as recomendações recentes na terapia da SDRA com a necessidade de ECMO. Uma das estratégias é a implementação de ECMO deve ser sugerida quando o cuidado respiratório convencional padrão citadas no decorrer do nosso artigo não corrigem a insuficiência respiratória. As principais indicações para ECMO são: (1) $\mathrm{PaO} 2$ / FiO2 $<100 \mathrm{mmHg}$, ou gradiente alvéolo-arterial da pressão parcial de oxigênio [P (A-a) O2]> $600 \mathrm{mmHg}$; (2) frequência ventilatória> 35 respirações por minuto, $\mathrm{pH}<7,2$ com pressão de platô> $30 \mathrm{cmH} 2 \mathrm{O}$; (3) Idade <65 anos; (4) ventilação mecânica <7 dias. Alternativamente, a ECMO para resgate de lesão pulmonar em ARDS grave, deve ser considerada se os pacientes atenderem a um dos seguintes critérios: (1) $\mathrm{PaO} 2$ / FiO2 <50 mmHg, mais de $3 \mathrm{~h}$; (2) $\mathrm{PaO} 2$ / FiO2 <80 mmHg, mais de $6 \mathrm{~h}$; (3) $\mathrm{pH}$ do sangue arterial $<7,25$ e PaCO2 $>60 \mathrm{mmHg}$, mais de $6 \mathrm{~h}$. Assim a implementação precoce de ECMO . Está comprovado que minimiza a pressão respiratória dirigida, inibe a inflamação pulmonar e sistêmica e reduz a disfunção grave do pulmão e dos órgãos extrapulmonares.

\section{CONCLUSÃO}

A ECMO veno-venosa ou venoarterial é uma modalidade terapêutica atualmente disponível para casos de infecção pelo Sars- CoV-2 que cursam com hipoxemia refratária ao suporte ventilatório padrão ou com SDRA grave que não respondem ao tratamento convencional. Neste contexto, em serviços de terapia intensiva foi possível observar taxas de mortalidade discretamente menores em pacientes com SDRA por infecção pelo COVID-19 que fizeram uso de ECMO, em comparação à terapia respiratória convencional.

A discordância sobre o uso da ECMO apenas em casos individualizados ou seu uso de forma irrestrita ainda persiste, tendo em vista a manutenção do questionamento acerca de prioridades, entre os diversos perfis de pacientes, no acesso ao tratamento. No entanto, a escassez de recursos impõe a priori o uso naqueles

RC: 92267

Disponível em: https://www.nucleodoconhecimento.com.br/saude/indicacoes-do-uso 
indivíduos que teriam algum ganho de expectativa de vida, visto que é uma terapêutica de alto custo, requer especialista apto a desenvolvê-la, equipamentos e disposição de leito em UTI.

\section{REFERÊNCIAS}

COMBES, Alain et al. Extracorporeal Membrane Oxygenation for Severe Acute Respiratory Distress Syndrome. N Engl J Med 2018; 378:1965-1975. Disponível em: $<$ https://www.nejm.org/doi/full/10.1056/NEJMoa1800385\#article_citing_articles>. Acesso em: 13 mai. 2021.

F, Argento et al. Membrana de oxigenación extracorpórea en infección por COVID19. Instituto de Efectividade Clinica y Sanitaria, Buenos Aires, v. 1, n. 1, p. 1-13, jul./2020. Disponível em: <https://docs.bvsalud.org/biblioref/2020/08/1116523/iecsirr-783-va-ecmo-en-covid-19.pdf>. Acesso em: 13 mai. 2021.

LI, Min et al. Extracorporeal membrane oxygenation support in 2019 novel coronavirus disease: indications, timing, and implementation. Chinese Medical Journal, China, v. 113, n. 9, p. 1115-1117, mai./2020. DOI: 10.1097/CM9.0000000000000778.

Disponível em: <https://journals.Iww.com/cmj/Fulltext/2020/05050/Extracorporeal_membrane_oxyge nation_support_in.18.aspx>. Acesso em: 13 mai. 2021.

MA, Xiaochun et al. Extracorporeal Membrane Oxygenation (ECMO) in Critically III Patients with Coronavirus Disease 2019 (COVID-19) Pneumonia and Acute Respiratory Distress Syndrome (ARDS). Med Sci Monit, China, v. 26, n. 925364, p. 1-10, ago./2020. DOI: 10.12659/MSM.925364. Disponível em: <https://www.medscimonit.com/abstract/index/idArt/925364>. Acesso em: 13 mai. 2021.

MACGREGOR, Robert et al. Extracorporeal Membrane Oxygenation for Pediatric Patients With Coronavirus Disease 2019-Related Illness. Pediatric Critical Care

RC: 92267

Disponível em: https://www.nucleodoconhecimento.com.br/saude/indicacoes-do-uso 
Medicine, Illinois, v. 21, n. 10, p. 893-897, out./2020. DOI: 10.1097/PCC. 0000000000002432 . $<$ https://journals.Iww.com/pccmjournal/Fulltext/2020/10000/Extracorporeal_Membran e_Oxygenation_for_Pediatric.5.aspx>. Acesso em: 13 mai. 2021.

\section{MINISTÉRIO DA SAÚDE. Oxigenação por membrana extracorpórea (ECMO)} para tratamentos de pacientes com COVID-19. Disponível em: $<$ https://docs.bvsalud.org/biblioref/2020/05/1096170/ecmo-covid19.pdf>. Acesso em 28 mai. 2021.

PARHAR, Ken Kuljit et al. Optimizing provision of extracorporeal life support during the COVID-19 pandemic: practical considerations for Canadian jurisdictions. Journal of the Canadian Medical Association, Canadá, v. 192, n. 14, p. 372-374, mar./2020. DOI: 10.1503/cmaj.200448. Disponível em: <https://www.cmaj.ca/content/192/14/E372>. Acesso em: 13 mai. 2021.

PEEK, Giles J. et al. Efficacy and economic assessment of conventional ventilatory support versus extracorporeal membrane oxygenation for severe adult respiratory failure (CESAR): a multicentre randomised controlled trial. Lancet (London, England) vol. 374,9698 (2009): 1351-63. doi:10.1016/S0140-6736(09)61069-2

QU, Jie-Ming et al. Guidance for the management of adult patients with coronavirus disease 2019: subtítulo do artigo. Chinese Medical Journal, China, v. 133, n. 13, p. 1575-1594, jul./2020. DOI: 10.1097/CM9.0000000000000899. Disponível em: $<$ https://journals.Iww.com/cmj/Fulltext/2020/07050/Guidance_for_the_management of_adult_patients_with.10.aspx>. Acesso em: 13 mai. 2021.

RAMAN, Lakshmi et al. Extracorporeal Life Support Organization Coronavirus Disease 2019 Interim Guidelines: A Consensus Document from an International Group of Interdisciplinary Extracorporeal Membrane Oxygenation Providers. ASAIO Journal, Dallas, v. $66, \quad$ n. 7 , $\quad$ p. 707-721, abr./2020. DOI: 10.1097/MAT.0000000000001193.

Disponível

em:

RC: 92267

Disponível em: https://www.nucleodoconhecimento.com.br/saude/indicacoes-do-uso 
$<$ https://journals.Iww.com/asaiojournal/Fulltext/2020/07000/Extracorporeal_Life_Supp ort_Organization.1.aspx>. Acesso em: 13 mai. 2021.

RAMANATHAN, Kollengode et al. Planning and provision of ECMO services for severe ARDS during the COVID-19 pandemic and other outbreaks of emerging infectious diseases. The Lancet Respiratory Medicine, Nova York, v. 8, n. 5, p. 518-526, mar./2020. DOI: https://doi.org/10.1016/S2213-2600(20)30121-1. Disponível em: <https://www.thelancet.com/journals/lanres/article/PIIS22132600(20)30121-1/fulltext>. Acesso em: 13 mai. 2021.

SULTAN, Ibrahim. The role of extracorporeal life support for patients with COVID-19: Preliminary results from a statewide experience. Journal of Cardiac Surgery, Pittsburgh, v. 35, n. 7, p. 1410-1413, jul./2020. DOI: 10.1111/jocs.14583. Disponível em: <https://onlinelibrary.wiley.com/doi/10.1111/jocs.14583>. Acesso em: 13 mai. 2021

WIEDEMANN, Dominik et al. Recommendations for extracorporeal membrane oxygenation (ECMO) in COVID-19 patients: subtítulo do artigo. Wiener klinische Wochenschrift: The Central European Journal of Medicine, Viena, v. 132, n. 21, p. 671-676, jul./2020. DOI: https://doi.org/10.1007/s00508-020-01708-8. Disponível em: <https://link.springer.com/article/10.1007\%2Fs00508-020-01708-8>. Acesso em: 13 mai. 2021.

YAN, Xiao et al. Extracorporeal Membrane Oxygenation for Coronavirus Disease 2019-Induced Acute Respiratory Distress Syndrome: A Multicenter Descriptive Study. Critical Care Medicine, Mount Prospect, v. 48, n. 9, p. 1289-1295, set./2020. DOI: 10.1097/CCM.0000000000004447. em: <https://journals.Iww.com/ccmjournal/Fulltext/2020/09000/Extracorporeal_Membrane _Oxygenation_for.6.aspx>. Acesso em: 13 mai. 2021.

Enviado: Julho, 2021.

RC: 92267

Disponível em: https://www.nucleodoconhecimento.com.br/saude/indicacoes-do-uso 
Aprovado: Julho, 2021.

RC: 92267

Disponível em: https://www.nucleodoconhecimento.com.br/saude/indicacoes-do-uso 